\title{
Statistical Properties of the Error Covariance in a Kalman Filter with Random Measurement Losses
}

\author{
Eduardo Rohr, Damián Marelli and Minyue Fu
}

\begin{abstract}
In this paper we study statistical properties of the error covariance matrix of a Kalman filter, when it is subject to random measurement losses. We introduce a sequence of tighter upper bounds for the asymptotic expected error covariance (EEC). This sequence starts with a given upper bound in the literature and converges to the actual asymptotic EEC. Although we have not yet shown the monotonic convergence of this whole sequence, monotonic convergent subsequences are identified. The feature of these subsequences is that a tighter upper bound is guaranteed if more computation is allowed. An iterative algorithm is provided for computing each of these upper bounds. A byproduct of this paper is a more compact proof for a known necessary condition on the measurement arrival probability for the asymptotic EEC to be finite. A similar analysis leads to a necessary condition on the measurement arrival probability for the error covariance to have a finite asymptotic variance.
\end{abstract}

\section{INTRODUCTION}

The low cost and high flexibility of networked systems has encouraged its use in wide areas, including control systems where information must be shared between sensors, controllers, and actuators. Under the control systems' perspective, this new technology has imposed new challenges, concerning how to deal with the effects of quantisation, delays and loss of packets, leading to the development of a new networked control theory [1].

The study of state estimators when measurements are subject to random delays and losses finds its application in both control and signal processing. Most estimators are based on the well-known Kalman filter [2]. In order to cope with network induced effects, the standard Kalman filter paradigm needs to undergo modifications. In this paper we consider this problem in the case of random missing measurements.

In order to cope with missing measurements, the update equation of the Kalman filter is dependent on whether a measurement arrives or not. When a measurement is available, the filter performs the standard update equation. On the other hand, if the measurement is missing, it must produce an open loop estimation, which as pointed out in [3], can be interpreted as the standard update equation when the measurement noise is infinite. The knowledge of the expected error covariance (EEC) of a state estimator is important to assess the performance of the estimator. Besides that, a clear understanding of how the system parameters and network delivery rates affects the EEC, permits a better system design, where the tradeoff between conflicting interests must be evaluated.

The authors are with the School of Electrical Engineering and Computer Science, University of Newcastle, University Drive, Callaghan NSW 2308, Australia. E-mail: eduardo.rohr@uon.edu.au
Studies on how to compute the EEC can be dated back at least to [4], where upper and lower bounds for the EEC were obtained by using a constant gain on the estimator. In [3], the same upper bound was derived as the limiting value of a recursive equation that computes a weighted average of the next possible error covariances. A similar result which allows partial observation losses was presented in [5]. In [6], [7], it is showed that a system in which the sensor sends the state estimate instead of the raw measurement will provide a better error covariance. However, this scheme requires the use of more complex sensors.

In this paper we study the limit (i.e., asymptotic) value of the EEC for a discrete-time system, in which the arrival of a measurement is a Bernoulli process with known probability. As the exact calculation of the limit EEC requires very large computational resources, we introduce a sequence of upper bounds for this matrix. The properties of this sequence are that its first element equals the upper bound stated in [3], and the sequence converges to the limit EEC. We also identify subsequences of this sequence which convergence to the limit EEC monotonically. Each element of this sequence is the solution of a non-linear equation, which does not involve the previous elements of the sequence, but whose numerical evaluation increases with the sequence index. We show that recursively evaluating this equation leads monotonically to its solution. Numerical experiments show that the first few elements of this sequence provide upper bounds which are much tighter than the bound stated in [3].

The authors of [3] also stated a condition on the measurement arrival probability, which is necessary for the EEC to have a finite limit value. We give an alternative derivation of this result, which not only is more compact, but also permits deriving a similar condition to study the limit value of the variance of the error covariance. This is a relevant study since in most applications it is desirable to have both finite mean and finite asymptotic variance for the error covariance.

The rest of the paper is organized as follows. In Section II we formulate the state estimation problem in the presence of random measurements losses. In Section III we derive the aforementioned sequence of upper bounds for the EEC, and give a monotonically convergent subsequence. In Section IV we provide an iterative algorithm for computing the elements of this sequence. In Section $\mathrm{V}$ we study conditions on the measurement arrival probability which are necessary for the error covariance matrix to have finite asymptotic mean and variance. In Section VI we present the numerical experiments. Concluding remarks are reached in Section VII. 


\section{Problem Statement}

Consider the discrete-time linear system:

$$
\begin{cases}x_{t+1} & =A x_{t}+w_{t} \\ y_{t} & =C x_{t}+v_{t}\end{cases}
$$

where $x \in \Re^{n}$ is the state having initial value $x_{0} \sim N\left(0, P_{0}\right)$ with $P_{0}>0, y \in \Re^{p}$ is the measurement process, $w \sim$ $N(0, Q)$ with $Q \geq 0$ is the process noise and $v \sim N(0, R)$ with $R \geq 0$ is the measurement noise. The measurements are sent to an estimator through a network subject to random packet losses, but without delays. Let $\gamma_{t}$ be a binary random variable describing the arrival of a measurement at time $t$. We define that $\gamma_{t}=1$ when $y_{t}$ was received at the estimator and $\gamma_{t}=0$ otherwise. We also assume that $\gamma_{t}$ is independent of $\gamma_{s}$ for $t \neq s$. The probability to receive a measurement is given by

$$
\lambda=\mathcal{P}\left(\gamma_{t}=1\right) .
$$

Let $P_{t}$ be the error covariance matrix (or error covariance for short) of the state estimate at time $t$, which is updated according to

$P_{t+1}= \begin{cases}\Phi_{1}\left(P_{t}\right)=A P_{t} A^{\prime}+Q-A K_{t} C P_{t} A^{\prime} & , \gamma_{t}=1 \\ \Phi_{0}\left(P_{t}\right)=A P_{t} A^{\prime}+Q & , \gamma_{t}=0\end{cases}$

with $K_{t}=P_{t} C^{\prime}\left(C P_{t} C^{\prime}+R\right)^{-1}$. Let $S_{m}^{N}, m=1, \ldots, 2^{N}$ be the set of all possible binary sequences of length $N$. We define the function $\phi\left(P_{t}, S_{m}^{N}\right)$ as the update of $P_{t}$ according to the sequence $S_{m}^{N}=\left\{\gamma_{t}, \gamma_{t+1}, \ldots, \gamma_{t+N-1}\right\}$ of measurement arrivals, i.e.,

$$
P_{t+N}=\phi\left(P_{t}, S_{m}^{N}\right)=\Phi_{\gamma_{t+N-1}} \circ \Phi_{\gamma_{t+N-2}} \circ \ldots \Phi_{\gamma_{t}}\left(P_{t}\right)
$$

where $\circ$ denotes the composition of functions (i.e. $f \circ g(x)=$ $f(g(x)))$.

Using (4), with $t=0$, we can compute the exact error covariance matrix $P_{N}$ for a given known arrival sequence. When this sequence is formed by random variables, $P_{N}$ becomes a random matrix. In this case, we are interested in computing the expected error covariance (EEC). To do so, we average the error covariances for all possible arrival sequences, according to their probabilities, i.e.,

$$
\bar{P}_{N}=E\left\{P_{N}\right\}=\sum_{m=1}^{2^{N}} \phi\left(P_{0}, S_{m}^{N}\right) \mathcal{P}\left(S_{m}^{N}\right),
$$

where $\mathcal{P}\left(S_{m}^{N}\right)=\lambda^{\Lambda}(1-\lambda)^{N-\Lambda}$ is the probability of the arrival sequence $S_{m}^{N}$, with $\Lambda=\sum_{p=0}^{N-1} \gamma_{p}$ being the number of measurements received between instants 0 and $N-1$. As $N$ tends to infinity, the EEC tends to its limit value

$$
\bar{P}=\lim _{N \rightarrow \infty} \bar{P}_{N} .
$$

It was shown in [3] that, if the system (1) is unstable, detectable, and the pair $\left(A, Q^{1 / 2}\right)$ is controllable, there exists a constant $0 \leq \lambda_{1} \leq 1$, called the critical measurement arrival probability, such that $\bar{P}<\infty$ if and only if $\lambda>\lambda_{1}$. Moreover, the limit $\bar{P}$ is independent of $P_{0}$.

The direct computation of (6) is impractical, since its complexity grows exponentially with $N$. To cope with this, we introduce below a method for deriving a sequence of upper bounds for $\bar{P}$, which converges to $\bar{P}$ on the limit.

\section{A Sequence of Upper Bounds fOr the EEC}

We state below two Lemmas that will be used to prove the main results of this section, namely, Theorems 2 and 1.

Lemma 1: The following statements hold for the functions $\Phi_{0}(\cdot)$ and $\Phi_{1}(\cdot)$ defined in (3):

a) If $X_{i} \geq 0$ and $\lambda_{i} \geq 0$ for $i=1,2, \cdots, I$ and $\sum_{i=1}^{I} \lambda_{i}=1$, then

$$
\begin{aligned}
& \Phi_{0}\left(\sum_{i=1}^{I} \lambda_{i} X_{i}\right)=\sum_{i=1}^{I} \lambda_{i} \Phi_{0}\left(X_{i}\right) \\
& \Phi_{1}\left(\sum_{i=1}^{I} \lambda_{i} X_{i}\right) \geq \sum_{i=1}^{I} \lambda_{i} \Phi_{1}\left(X_{i}\right)
\end{aligned}
$$

b) If $Y \geq X$, then

$$
\begin{aligned}
& \Phi_{0}(Y) \geq \Phi_{0}(X) \\
& \Phi_{1}(Y) \geq \Phi_{1}(X)
\end{aligned}
$$

Proof: The properties (7) and (9) are straightforward because $\Phi_{0}(X)$ is affine in $X$. The proof of (8) follows from the concavity of $\Phi_{1}(X)$; see [3, Lemma 1e]. The proof of (10) follows from [3, Lemma 1c].

Lemma 2: Consider the operator

$$
g_{N}(X)=\sum_{m=1}^{2^{N}} \phi\left(X, S_{m}^{N}\right) \mathcal{P}\left(S_{m}^{N}\right) .
$$

Then, for any $N, M \in \mathbb{N}$ and $X \geq 0$,

$$
g_{N+M}(X) \leq g_{N}\left(g_{M}(X)\right) .
$$

Also, if $Y \geq X$, then

Proof: From (11), we have

$$
g_{N}(Y) \geq g_{N}(X) .
$$

$$
\begin{aligned}
& g_{N}\left(g_{M}(X)\right)= \\
= & \sum_{n=1}^{2^{N}} \mathcal{P}\left(S_{n}^{N}\right) \phi\left(\left[\sum_{m=1}^{2^{M}} \mathcal{P}\left(S_{m}^{M}\right) \phi\left(X, S_{m}^{M}\right)\right], S_{n}^{N}\right) .
\end{aligned}
$$

Now, $\phi\left(\cdot, S_{n}^{N}\right)$ is the composition of a sequence of functions, each one being either $\Phi_{0}$ or $\Phi_{1}$. Let $\Phi_{i}$ be the first term of that sequence, i.e., $\phi\left(\cdot, S_{n}^{N}\right)=\psi \circ \Phi_{i}(X)$. Then, using Lemma 1-a) and interpreting $\mathcal{P}\left(S_{m}^{M}\right)$ as $\lambda_{i}$, (14) becomes

$$
\begin{aligned}
& g_{N}\left(g_{M}(X)\right) \geq \\
& \quad \sum_{n=1}^{2^{N}} \mathcal{P}\left(S_{n}^{N}\right) \psi\left(\sum_{m=1}^{2^{M}} \mathcal{P}\left(S_{m}^{M}\right) \Phi_{i} \circ \phi\left(X, S_{m}^{M}\right)\right) .
\end{aligned}
$$

Repeating the same operation for each element in the remaining sequence $\psi$, we obtain

$$
\begin{aligned}
& g_{N}\left(g_{M}(X)\right) \geq \\
\geq & \sum_{n=1}^{2^{N}} \mathcal{P}\left(S_{n}^{N}\right) \sum_{m=1}^{2^{M}} \mathcal{P}\left(S_{m}^{M}\right) \phi\left(\phi\left(X, S_{m}^{M}\right), S_{n}^{N}\right) \\
= & g_{N+M}(X) .
\end{aligned}
$$


To show (13), notice that $g_{N}(X)$ is a weighted sum of terms, each of which is a composition sequence of $\Phi_{0}$ and $\Phi_{1}$. Then, te result follows from Lemma 1-b).

We now define

$$
\hat{P}_{N}:=\operatorname{sol}_{X}\left\{X=g_{N}(X)\right\}
$$

i.e., $\hat{P}_{N}=g_{N}\left(\hat{P}_{N}\right)$. In particular, for $N=1$, we have

$$
\hat{P}_{1}=A \hat{P}_{1} A^{\prime}+Q-\lambda A \hat{P}_{1} C^{\prime}\left(C \hat{P}_{1} C^{\prime}+R\right)^{-1} C \hat{P}_{1} A^{\prime}
$$

which is the upper bound for the EEC stated in [3]. It is clear from (5), (6) and (11) that $\hat{P}_{N} \rightarrow \bar{P}$ as $N \rightarrow \infty$. The next theorem formally states this fact, as well as the existence of sub-sequences of $\hat{P}_{N}$ whose convergence to $\bar{P}$ is monotonic.

Theorem 1: If $\lambda_{1}<\lambda \leq 1$ (where $\lambda_{1}$ is the critical probability mentioned earlier), and the condition of Theorem 2 in the next section holds, then

$$
\lim _{N \rightarrow \infty} \hat{P}_{N}=\bar{P},
$$

and, for any $c, N \in \mathbb{N}$

$$
\bar{P} \leq \hat{P}_{c N} \leq \hat{P}_{N} \leq \hat{P}_{1}
$$

Proof: From Lemma 2, we have that

$$
g_{c N}(X) \leq g_{N} \circ g_{(c-1) N}(X) \leq \ldots g_{N}^{c}(X)
$$

where the superscript $c$ denotes the composition operation $c$ times. Now, from (13) and (19), it can be verified that

$$
g_{c N}^{k}(X) \leq g_{N}^{c k}(X)
$$

Using Theorem 2 in the next section, $\lim _{k \rightarrow \infty} g_{c N}^{k}(X)$ and $\lim _{k \rightarrow \infty} g_{N}^{k}(X)$ converge for any $X>0$. Using this, (20) and (15), we have

$$
\hat{P}_{c N}=\lim _{k \rightarrow \infty} g_{c N}^{k}(X) \leq \lim _{k \rightarrow \infty} g_{N}^{c k}(X)=\hat{P}_{N} .
$$

While Theorem 1 states that the convergence of the sequence $\hat{P}_{N}$ to $\bar{P}$ is only monotonic on sub-sequences, numerical experiments suggest that the whole sequence $\hat{P}_{N}$ converges monotonically to $\bar{P}$. Hence, we conjecture that this holds in general. However, we were not able to prove this property yet.

\section{Computing The EEC Bounds}

In this section we study the numerical computation of the EEC bounds (15). For a given $N, \hat{P}_{N}$ can be recursively computed as follows:

$$
\hat{P}_{N}^{(k+1)}=\sum_{m=1}^{2^{N}} \phi\left(\hat{P}_{N}^{(k)}, S_{m}^{N}\right) \mathcal{P}\left(S_{m}^{N}\right) .
$$

It is clear that, when the recursions (22) converge to an equilibrium point, this must be a solution (15), i.e., $\hat{P}_{N}=$ $\lim _{k \rightarrow \infty} P_{N}^{(k)}$. Theorem 2 below states the condition under which the recursions (22) are guaranteed to converge to $\hat{P}_{N}$, for any initial condition $\hat{P}_{N}^{(1)}$. Also, it follows from (13) that this convergence is monotonic.
Theorem 2: For any matrix $M$ and $X \geq 0$, let

$$
\begin{aligned}
& \tilde{\Phi}_{0, M}(X)=\Phi_{0}(X) \\
& \tilde{\Phi}_{1, M}(X)=(A+M C) X(A+M C)^{\prime}+Q+M R M^{\prime}
\end{aligned}
$$

Also, for a sequence $\mathbf{m}=M_{1}, \cdots, M_{N}$ of matrices, and a sequence of binary coefficients $S^{N}=\gamma_{1}, \cdots, \gamma_{N}$, we define $\tilde{\phi}\left(X, S^{N}, \mathbf{m}\right)=\tilde{\Phi}_{\gamma_{N}, M_{N}} \circ \cdots \circ \tilde{\Phi}_{\gamma_{1}, M_{1}}(X)$, and for a collection $\mathcal{M}=\left\{\mathbf{m}_{m}: m=1, \ldots, 2^{N}\right\}$ of matrix sequences, we define

$$
\tilde{g}_{N}(X, \mathcal{M})=\sum_{m=1}^{2^{N}} \tilde{\phi}\left(X, S_{m}^{N}, \mathbf{m}_{m}\right) \mathcal{P}\left(S_{m}^{N}\right) .
$$

Then, if there exists $X \geq 0$ and a collection of matrix sequences $\mathcal{M}$ such that $X \leq \tilde{g}_{N}(X, \mathcal{M})$, then the recursions (22) are guaranteed to converge to $\hat{P}_{N}$, for any initial condition $\hat{P}_{N}^{(1)}$

Proof: [Sketch version] The result was shown for the case $N=1$ in [3, Theorem 1]. The proof for the general case follows the same argument. Due to space limitations, we only provide here a sketch of it.

Let $V^{(k+1)}=g_{N}\left(V^{(k)}\right)$, with $V^{(1)}=0$. From (13), it follows that the sequence $0=V^{(1)} \leq V^{(2)} \leq V^{(3)} \ldots$ is increasing. Also, following the argument of Lemma 4 in [3], it can be shown that this sequence is bounded, so it must converge to a limit value $P^{\star}$, i.e.,

$$
\lim _{k \rightarrow \infty} V^{(k)}=P^{\star} .
$$

Now, it is straightforward to verify that, $\tilde{\Phi}_{1, M_{X}}(X)=$ $\Phi_{1}(X)$, where $M_{X}=-A X C^{\prime}\left(C X C^{\prime}+R\right)^{-1}$. Using this, it follows that $\tilde{g}_{N}\left(X, \mathcal{M}_{X}\right)=g_{N}(X)$, if $\mathcal{M}_{X}$ denotes the "right" collection of matrix sequences. Also, from Lemma 1b) in [3], $\tilde{\Phi}_{1, M_{X}}(X) \leq \tilde{\Phi}_{1, M}(X)$ for a general $M$, and therefore, it can be verified that, for a general collection of matrix sequences $\mathcal{M}$,

$$
\tilde{g}_{N}\left(X, \mathcal{M}_{X}\right) \leq \tilde{g}_{N}(X, \mathcal{M}) .
$$

For a given $M, \tilde{\Phi}_{1, M}(X)$ is an affine function of $X$, hence so is $\tilde{g}_{N}(X, \mathcal{M})$ for a given $\mathcal{M}$. Let

$$
\tilde{g}_{N}(X, \mathcal{M})=\tilde{l}_{N}(X, \mathcal{M})+\tilde{c}_{N}(\mathcal{M}),
$$

where $\tilde{l}_{N}(X, \mathcal{M})$ is linear in $X$ and $\tilde{c}_{N}(\mathcal{M})$ is a constant term depending on $\mathcal{M}$. Also, let $W^{(k+1)}=g_{N}\left(W^{k}\right)$, with $W^{(1)} \geq P^{\star}$. From (24), we have that

$$
\begin{aligned}
W^{(k+1)}-P^{\star} & =g_{N}\left(W^{(k)}\right)-g_{N}\left(P^{\star}\right) \\
& =\tilde{g}_{N}\left(W^{(k)}, \mathcal{M}_{W^{(k)}}\right)-\tilde{g}_{N}\left(P^{\star}, \mathcal{M}_{P^{\star}}\right) \\
& \leq \tilde{g}_{N}\left(W^{(k)}, \mathcal{M}_{P^{\star}}\right)-\tilde{g}_{N}\left(P^{\star}, \mathcal{M}_{P^{\star}}\right) \\
& =\tilde{l}_{N}\left(W^{(k)}-P^{\star}, \mathcal{M}_{P^{\star}}\right) .
\end{aligned}
$$

Since $g_{N}\left(P^{\star}\right)=P^{\star}$, it follows from (13) that

$$
W^{(k)} \geq P^{\star}, \text { for all } k \text {. }
$$

Also, using the argument of Lemma 3 in [3], it can be shown that $\lim _{k \rightarrow \infty} \tilde{l}_{N}^{k}\left(Y, \mathcal{M}_{P^{\star}}\right)=0$, whenever $Y>0$. Hence, from (26) and (25), we have that

$$
\lim _{k \rightarrow \infty} W^{(k)}=P^{\star} .
$$


Now, for any $P^{(1)} \geq 0$, put $W^{(1)}=P^{(1)}+P^{\star}$. From (13), it follows that $V^{(k)} \leq P^{(k)} \leq W^{(k)}$, for all $k$. We have that $\lim _{k \rightarrow \infty} V^{(k)}=P^{\star}$, and since $W^{(1)} \geq P^{\star}$, $\lim _{k \rightarrow \infty} W^{(k)}=P^{\star}$. Hence, $\lim _{k \rightarrow \infty} P^{(k)}=P^{\star}=\hat{P}_{N}$.

\section{ReQuirements on the MeAsurement ARrival PROBABILITY}

In this section we study the requirements on the measurement arrival probability $\lambda$, so that the limit probability distribution of the error covariance matrix $P_{N}$ has finite mean and finite variance. In the case of finite mean, a lower bound on the required value of $\lambda$ was derived in [3]. We propose an alternative proof of this result, which is significantly more compact, and its argument can be easily modified to derive a similar bound for the value of $\lambda$ required to have a finite variance.

\section{A. For the error covariance matrix to have finite mean}

It was shown in [3] that a necessary condition for the EEC $\bar{P}_{N}$ in (5) to have a finite limit value, is that the measurement arrival probability $\lambda$ is smaller than a given constant $\lambda_{1}$. In this section we provide an alternative proof of that result. For simplicity, we assume that the matrix $A$ is diagonalizable and unstable.

Theorem 3: If $A$ is diagonalizable and unstable and $\lambda<$ $\underline{\lambda_{1}}$, then $\lim _{N \rightarrow \infty} \bar{P}_{N}=\infty$, where

$$
\underline{\lambda_{1}}=1-\frac{1}{\max |\operatorname{eig}(A)|^{2}}
$$

and $\max (|\operatorname{eig}(A)|)$ denotes the absolute value of the largest eigenvalue of $A$.

Proof: In order for the summation in (5) to be finite, each term must be finite. The term corresponding to all measurements being lost, is given by

$\phi\left(P_{0}, S_{1}^{N}\right) \mathcal{P}\left(S_{1}^{N}\right)=(1-\lambda)^{N}\left(A^{N} P_{0} A^{N}+\sum_{j=0}^{N-1} A^{j} Q A^{\prime j}\right)$.

Let $\sigma_{0}>0$ denote the smallest eigenvalue of $P_{0}$. Since $P_{0}>0$ and $Q \geq 0$, it follows that

$$
\phi\left(P_{0}, S_{1}^{N}\right) \mathcal{P}\left(S_{1}^{N}\right) \geq \sigma_{0}(1-\lambda)^{N}\left(A^{N} A^{N}\right) .
$$

Now, since $A$ is diagonalizable, we can write $A=M D M^{-1}$, with $D$ being a diagonal matrix containing the eigenvalues of $A$, and $M$ being an invertible matrix containing the corresponding eigenvectors as columns. Then, we have that

$$
\begin{aligned}
A^{N} A^{\prime N} & =M D^{N} M^{-1} M^{\prime-1} D^{N} M^{\prime} \\
& \geq \min \left|\operatorname{eig}\left(M^{-1} M^{\prime-1}\right)\right| M D^{2 N} M^{\prime} \\
& =\frac{1}{\max \left|\operatorname{eig}\left(M^{\prime} M\right)\right|} M D^{2 N} M^{\prime} \\
& =\frac{1}{\|M\|^{2}} M D^{2 N} M^{\prime}
\end{aligned}
$$

with $\|M\|$ being defined as the largest singular value of $M$. From (28) and (29), it follows that the largest eigenvalue $\sigma_{\max }$ of $\phi\left(P_{0}, S_{1}^{N}\right) \mathcal{P}\left(S_{1}^{N}\right)$ satisfies

$$
\begin{aligned}
\sigma_{\max } & \geq \frac{\sigma_{0}(1-\lambda)^{N}}{\|M\|^{2}} \max \left|\operatorname{eig}\left(M D^{2 N} M^{\prime}\right)\right| \\
& =\frac{\sigma_{0}(1-\lambda)^{N}}{\|M\|^{2}}\left\|M D^{N}\right\|^{2} \\
& \geq \frac{\sigma_{0}(1-\lambda)^{N}}{\|M\|^{2}\left\|M^{-1}\right\|^{2}}\left\|D^{N}\right\|^{2} \\
& =\frac{\sigma_{0}(1-\lambda)^{N}}{\|M\|^{2}\left\|M^{-1}\right\|^{2}} \max |\operatorname{eig}(A)|^{2 N} .
\end{aligned}
$$

Now, as $N \rightarrow \infty$, we have that

$$
(1-\lambda) \max |\operatorname{eig}(A)|^{2}<1
$$

is a condition which is necessary for $M_{N}^{(2)}$ to be finite, and the result follows.

\section{B. For the error covariance matrix to have finite variance}

Our study so far concerned the expected value of the error covariance matrix. The importance of this expected value is relative to its associated variance, as a big variance will render the knowledge of the expected value meaningless. Motivated by this, we parallel Section V-A to state a necessary condition on $\lambda$ so that the sum of the variances of all the entries of the error covariance matrix has finite asymptotic value. Notice that this condition turns out to be stronger than the one stated in Theorem 3.

Let $M_{N}^{(2)}$ denote the sum of the second moments of all the entries of the error covariance, i.e.,

$$
M_{N}^{(2)} \triangleq E\left\{\operatorname{trace}\left(P_{N} P_{N}^{\prime}\right)\right\}
$$

The next theorem states a condition on $\lambda$ which is necessary for $M_{N}^{(2)}$ to have a finite limit value.

Theorem 4: If $A$ is diagonalizable and unstable and $\lambda<$ $\underline{\lambda_{2}}$, then $\lim _{N \rightarrow \infty} M_{N}^{(2)}=\infty$, where

$$
\underline{\lambda_{2}}=1-\frac{1}{\max |\operatorname{eig}(A)|^{4}} \text {. }
$$

Proof: The proof is similar to the one in Theorem 3, and uses the same notation. From (31), we have that

$$
\begin{aligned}
M_{N}^{(2)} & =\sum_{m=1}^{2^{N}} \mathcal{P}\left(S_{m}^{N}\right) \operatorname{trace}\left(\phi\left(P_{0}, S_{m}^{N}\right) \phi^{\prime}\left(P_{0}, S_{m}^{N}\right)\right) \\
& \geq(1-\lambda)^{N} \operatorname{trace}\left(\phi\left(P_{0}, S_{1}^{N}\right) \phi^{\prime}\left(P_{0}, S_{1}^{N}\right)\right) \\
& \geq \sigma_{0}(1-\lambda)^{N} \operatorname{trace}\left(A^{N} A^{\prime N} A^{N} A^{\prime N}\right) \\
& \geq \sigma_{0}(1-\lambda)^{N} \max \left|\operatorname{eig}\left(A^{N} A^{N} A^{N} A^{\prime N}\right)\right| \\
& =\sigma_{0}(1-\lambda)^{N} \max \left|\operatorname{eig}\left(A^{N} A^{\prime N}\right)\right|^{2} \\
& \geq \frac{\sigma_{0}(1-\lambda)^{N}}{\|M\|^{4}\left\|M^{-1}\right\|^{4}} \max |\operatorname{eig}(A)|^{4 N}
\end{aligned}
$$

where the last step follows from the argument in the proof of Theorem 3. Taking the limit when $N \rightarrow \infty$, we have that

$$
(1-\lambda) \max |\operatorname{eig}(A)|^{4}<1
$$




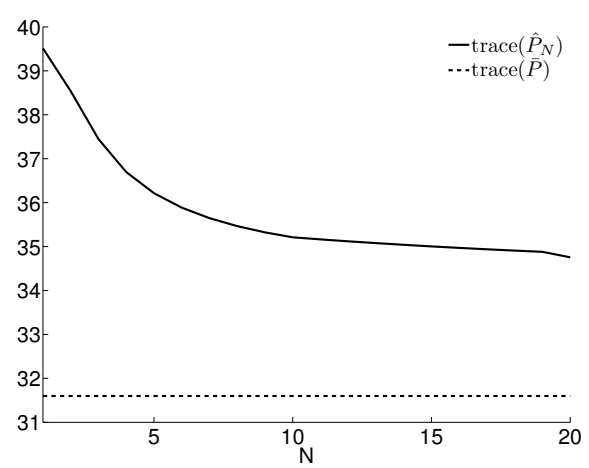

Fig. 1. Convergence of $\operatorname{trace}\left(\hat{P}_{N}\right)$ to $\operatorname{trace}(\bar{P})$ in the system (35).

is a condition which is necessary for $M_{N}^{(2)}$ to be finite, and the result follows.

It is easy to see that $\underline{\lambda_{2}} \geq \underline{\lambda_{1}}$.

Now, let $\sigma_{N}^{2}$ denote the sum of the variances of all the entries of the error covariance matrix, i.e.,

$$
\sigma_{N}^{2} \triangleq E\left\{\operatorname{trace}\left(\left(P_{N}-\bar{P}_{N}\right)\left(P_{N}-\bar{P}_{N}\right)^{\prime}\right)\right\} .
$$

It is straightforward to verify that

$$
\sigma_{N}^{2}=M_{N}^{(2)}-\operatorname{trace}\left(\bar{P}_{N} \bar{P}_{N}^{\prime}\right) .
$$

Therefore, if $\lambda<\underline{\lambda_{2}}$, then either the mean $\bar{P}_{N}$ of the EC, or its variance $\sigma_{N}^{2}$ or both will be infinite. In any of these cases, the knowledge of the EEC $\bar{P}_{N}$ will be meaningless.

\section{NUMERICAL EXPERIMENTS}

In this section we present some numerical experiments illustrating the results on the paper. To this end, we use the same system models used in [3].

\section{A. Convergence of the sequence $\hat{P}_{N}$ towards $\bar{P}$}

In the first experiment we study how the elements of the sequence of EEC bounds $\hat{P}_{N}$, approach the limit EEC value $\bar{P}$. We compute each element of the sequence using (22). To compute the limit EEC value, we average the correlation martices of $100 \times 10^{3}$ systems, after reaching their steady state. We also compare the upper bounds $\hat{P}_{N}$, with the first element $\hat{P}_{1}$, which is the bound considered in [3].

In Figure 1 we show the trace of the EEC bounds, as a function of $N$, for the system given by

$$
A=-1.25 \quad C=1 \quad R=2.5 \quad Q=1 .
$$

A particular property of this system is that $\mathrm{C}$ is invertible. Its critical arrival probability is $\lambda_{1}=0.36$, so we choose $\lambda=0.4$ to guarantee a finite EEC on the limit. We see that the convergence of trace $\left(\hat{P}_{N}\right)$ to trace $(\bar{P})$ is monotonic, and that the improvement of $\hat{P}_{20}$ over $\hat{P}_{1}$ is $12 \%$.

Figure 2 shows the same experiment for the following system, which has a single unstable eigenvalue

$$
A=\left[\begin{array}{ccc}
1.25 & 1 & 0 \\
0 & 0.9 & 7 \\
0 & 0 & 0.6
\end{array}\right] \quad C^{\prime}=\left[\begin{array}{ccc}
1 & 0 & 2
\end{array}\right]
$$

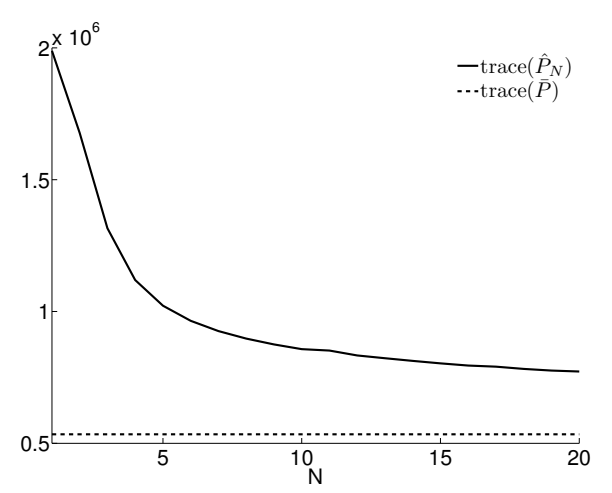

Fig. 2. Convergence of $\operatorname{trace}\left(\hat{P}_{N}\right)$ to trace $(\bar{P})$ in the system (36).

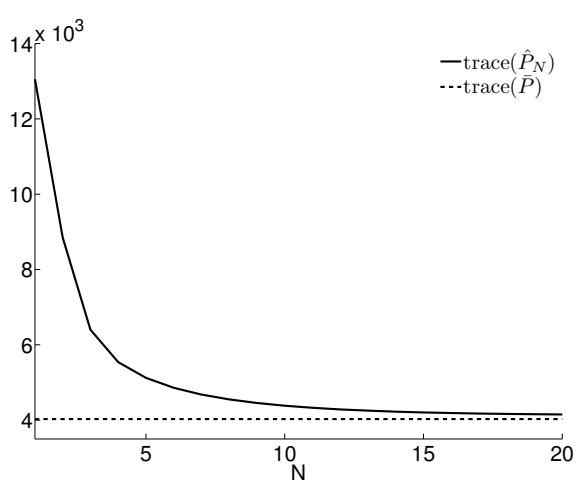

Fig. 3. Convergence of trace $\left(\hat{P}_{N}\right)$ to trace $(\bar{P})$ in the system (37).

The critical arrival probability for this system is also $\lambda_{1}=$ 0.36 , and again we choose $\lambda=0.4$. In this example, the improvement of $\hat{P}_{20}$ over $\hat{P}_{1}$ is $73 \%$.

In the last example, we study the system

$$
\begin{array}{cc}
A=\left[\begin{array}{cc}
1.25 & 0 \\
1 & 1.1
\end{array}\right] & C^{\prime}=\left[\begin{array}{ll}
1 & 1
\end{array}\right] \\
R=2.5 & Q=20 I
\end{array}
$$

which has two unstable eigenvalues. In this case, the value of $\lambda_{1}$ is not known, and we only know its inferior $\underline{\lambda_{1}}=0.36$ and superior $\overline{\lambda_{1}}=0.48$ bounds, and to guarantee a finite EEC on the limit we choose $\lambda=0.5$. The convergence of $\operatorname{trace}\left(\hat{P}_{N}\right)$ is shown in Figure 3, showing an improvement of $68 \%$, from $\hat{P}_{20}$ to $\hat{P}_{1}$. In this example we also observe that $\operatorname{trace}\left(\hat{P}_{20}\right)$ is a good approximation of $\operatorname{trace}(\bar{P})$.

We now study how the measurement arrival probability $\lambda$ affects the convergence rate of the sequence trace $\left(\hat{P}_{N}\right)$. We consider the system described by (36). Figure 4 shows the evolution of $\operatorname{trace}\left(\hat{P}_{N}\right) / \operatorname{trace}\left(\hat{P}_{1}\right)$ for different values of $\lambda$. We normalized the results (by dividing by trace $\left(\hat{P}_{1}\right)$ ) in order to facilitate the comparisons. Also, for each value of $\lambda$, we show in the legend of this figure the value $a=$ $(1-\lambda) \max (|\operatorname{eig}(A)|)^{2}$, since $a<1$ is a necessary criteria for the EEC to have a finite limit value (see (30)). We see that the convergence rate of trace $\left(\hat{P}_{N}\right)$ increases with an increase of $\lambda$. 


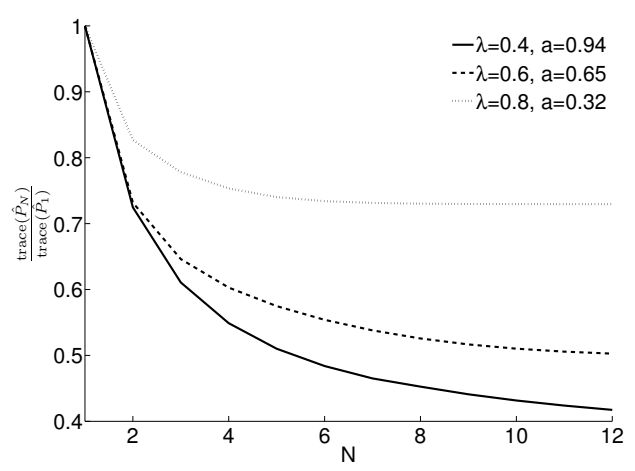

Fig. 4. Convergence rate of the sequence trace $\left(\hat{P}_{N}\right)$ for different measurement arrival probabilities.

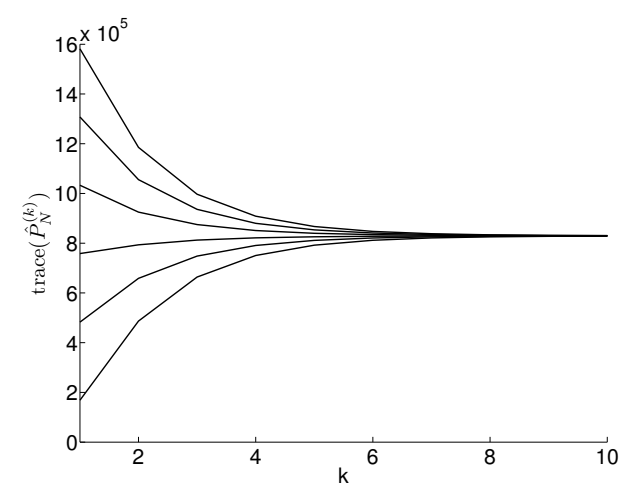

Fig. 5. Convergence of $\operatorname{trace}\left(\hat{P}_{N}^{(k)}\right)$ towards trace $\left(\hat{P}_{N}\right)$.

\section{B. Convergence of trace $\left(\hat{P}_{N}^{(k)}\right)$ towards trace $\left(\hat{P}_{N}\right)$}

In Section IV we stated an iterative formula which produces a sequence of matrices $\hat{P}_{N}^{(k)}$ which converges to $\hat{P}_{N}$ as $k \rightarrow \infty$, and we showed that this convergence is monotonic. We illustrate this property in Figure 5, where we consider the system described by (36), and we compute $\hat{P}_{12}$ using different initial values $\hat{P}_{12}^{(1)}$.

\section{Limit value of second order statistics}

We now illustrate the result presented in Theorem 4. Using (32), we find that if the meassure arrival probability $\lambda$ is smaller that $\lambda_{2}=0.59$, then either $\sigma_{N}^{2}$, as defined by (34), or the EEC $\bar{P}_{N}$, or both have infinite limit values. We use the system described by (36), for which $\lambda_{1}=0.36$. In Figure 6 we show the evolution of $\sigma_{N}^{2}$, for different values of $\lambda$. Using $\lambda=0.4$, which is enough to make the EEC finite, we see that $\sigma_{N}^{2}$ divergence very quickly. For $\lambda=0.58$, i.e., slightly below $\lambda_{2}$, the variance also diverges, but at a slower rate. Finally, for $\lambda=0.6$, i.e., slightly above $\underline{\lambda_{2}}, \sigma_{N}^{2}$ converges to a finite value, indicating that in this example the value $\lambda>\lambda_{2}$ is not only a necessary condition, but also an accurate sufficient condition for $\sigma_{N}^{2}$ to have a finite value in the limit.

\section{CONCLUSION}

In this paper we have studied some statistical properties of the error covariance matrix of the state estimator of a

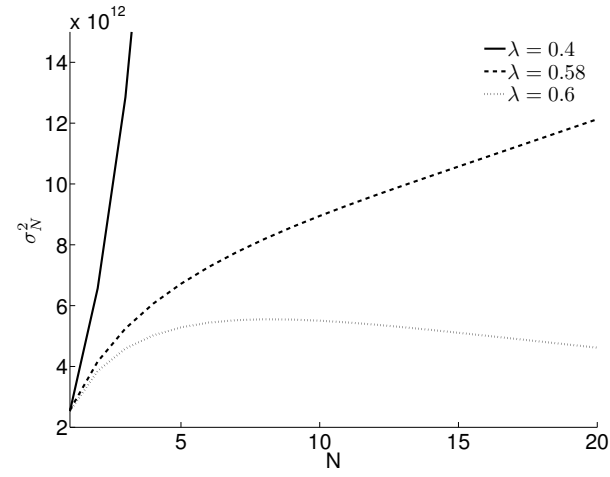

Fig. 6. Limit value of $\sigma_{N}^{2}$ for different measurement arrival probabilities.

dynamic system whose measurements are subject to random losses. We have proposed a sequence of upper bounds for the EEC which converges to the true EEC asymptotically. While we were not able to show the monotonic convergence of this sequence, we have provided sub-sequences for which this property holds. We have given a recursive algorithm to compute each term of this sequence, which converges monotonically to the solution. Experimental results show that the proposed upper bounds are significantly tighter than those reported in previous works.

Additionally, we have derived conditions on the measurement arrival probability which are necessary for the error covariance matrix to have finite mean and variance asymptotically. In the case of the mean, our derivation is an alternative proof of a similar condition which was reported previously. However, the argument of this proof is considerably more compact, and the same argument permits studying a similar necessary condition for the convergence of the variance.

\section{REFERENCES}

[1] L. Schenato, B. Sinopoli, M. Franceschetti, K. Poolla, and S.S. Sastry. Foundations of control and estimation over lossy networks. Proc .IEEE, 95(1):163, 2007.

[2] B.D.O. Anderson and J.B. Moore. Optimal filtering. Prentice-Hall Englewood Cliffs, NJ, 1979.

[3] B. Sinopoli, L. Schenato, M. Franceschetti, K. Poolla, M.I. Jordan, and S.S. Sastry. Kalman filtering with intermittent observations. IEEE Transactions on Automatic Control, 49(9):1453-1464, 2004.

[4] H M Faridani. Performance of kalman filter with missing measurements. Automatica, 22(1):117-120, 1986.

[5] X. Liu and A. Goldsmith. Kalman filtering with partial observation losses. IEEE Control and Decision, 2004.

[6] L. Schenato. Optimal estimation in networked control systems subject to random delay and packet drop. IEEE Transactions on Automatic Control, 53(5):1311-1317, 2008.

[7] A.F. Dana, V. Gupta, J.P. Hespanha, B. Hassibi, and R.M. Murray. Estimation over communication networks: Performance bounds and achievability results. American Control Conference, 2007. ACC '07, pages $3450-3455$, july 2007 . 